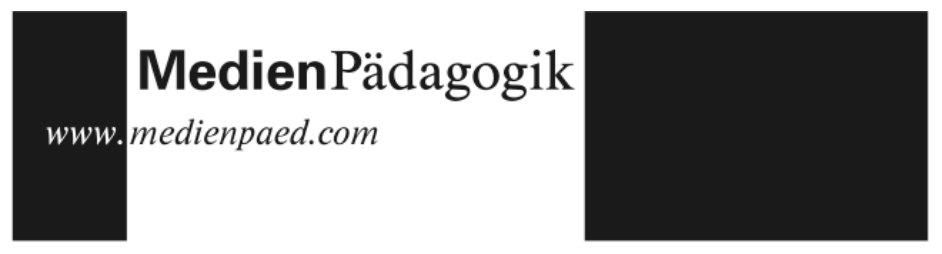

Rezensionen

Heinz Moser

Einführung in die

Medienpädagogik

Aufwachsen im Medienzeitalter

5., durchgesehene

und erweiterte Auflage

LERBUCH
Heinz Moser

Einführung in die Medienpädagogik.

Aufwachsen im Medienzeitalter

5., durchgesehene und erweiterte Auflage

Wiesbaden: VS Verlag für Sozialwissenschaften, 2010. 457 Seiten

ISBN: 978-3-531-16164-8

$€ 22.90$; CHF 27.30

\section{Aufwachsen im Medienzeitalter}

In seiner Einführung stellt Moser das Forschungs- und Handlungsfeld der Medienpädagogik aus der Perspektive einer mediatisierten Gesellschaft vor. Er geht grundsätzlich davon aus, dass Medien Sozialisations- und Lernprozesse heute selbstverständlich begleiten und für Kinder und Jugendliche vielfältige Funktionen erfüllen. In seiner kritisch-optimistischen (vgl. Süß 2004) und konsequent konstruktivistischen Argumentation setzt sich der Autor mit unterschiedlichen Positionen und Ansätzen auseinander und verdeutlicht, dass die Medienpädagogik individuelle und gesellschaftliche Medienbildungs- und Entwicklungsprozesse sehr differenziert betrachtet und zu unterstützen sucht.

Anhand markanter Medienereignisse zeigt Moser zu Beginn des Buches, dass mediale und gesellschaftliche Entwicklungen einander stets begleiten und dass Realität und Fiktion keinesfalls dichotomen Konzepte sondern fließende Konstruktionen sind. Er beleuchtet die Durchdringung des Realen mit Medientechnologien und Medienprodukten und kommt zu dem Schluss, dass sich durch technische wie inhaltliche Medieninnovationen die Bedeutungen von Zeit, Raum, Geschichte und Wahrheit immer wieder verändert haben. Das Aufwachsen in der Erlebnisgesellschaft versteht Moser gleichsam als Emanzipation der Kinder von bewahrpädagogischen Bemühungen. Diese Emanzipation spiegelt sich in verschiedenen gesellschaftlich relevanten Bereichen wider, neben den Medien auch in neuen Identitätskonstruktionen, Verinselung, zunehmender Mobilität, Beschleunigung etc. 
Moser entfaltet zunächst einen sehr informativen Überblick über wichtige diesbezügliche Erkenntnisse, wobei er sich vor allem auf die Entwicklung des kindlichen Fernsehverständnisses konzentriert. Daran anknüpfend diskutiert er verschiedene Studienergebnisse zu den Verarbeitungs- und Gebrauchsweisen von Fernsehinhalten. Intensiver in den Blick genommen wird dabei der häufig untersuchte Zusammenhang von Gewalt und fiktionalen Medieninhalten. Im Folgenden erweitert Moser den Blick auf die digitalen Medien und beschreibt Entwicklungen im Medienbereich (Medienkonvergenz) und auf Rezipientenseite (Digital Natives). Abschließend stellt Moser das Konzept der Medienkompetenz vor, übersetzt dieses in schulische Standards und Indikatoren und bindet es schließlich an den Begriff der Medienbildung. Im Schlussteil des Buches stehen die Veränderungen von Bildungsprozessen und Institutionen durch und mit Medien im Zentrum. Fazit ist die bildungstheoretische Notwendigkeit einer breiten Medienbildung.

Mosers «Einführung in die Medienpädagogik. Aufwachsen im Medienzeitalter» ist ein sehr informatives und aufschlussreiches Buch für an Argumentationszusammenhängen und Hintergründen der Medienpädagogik interessierte Leser/innen. Aufgrund der dominierenden kommunikationswissenschaftlichen Perspektive und der damit verbundenen ausführlichen Diskussion unterschiedlicher Ansätze und Positionierungen ist es jedoch weniger als eine grundlegende Einführung in Fragestellungen und Themen der Medienpädagogik zu sehen. So verliert das Buch nicht zuletzt durch seine vielen Detaillierungen zuweilen den intendierten einführenden Charakter.

Eine große Bereicherung sind die das Buch ergänzenden OnlineMaterialien. Die hier zur Verfügung gestellten Arbeitsaufgaben können Lernprozesse unterstützen, indem sie eine vertiefende Auseinandersetzung mit eigenen Erfahrungen auf der Grundlage des Buches herausfordern. Damit eignen sie sich nicht nur für das selbstorganisierte Lernen, sondern ebenso als didaktische Materialien für organisierte Lernprozesse.

Anja Klimsa

\section{Literatur}

Süss, Daniel. Mediensozialisation von Heranwachsenden. Dimensionen,

Konstanten, Wandel. Wiesbaden: VS Verlag für Sozialwissenschaften, 2004. 